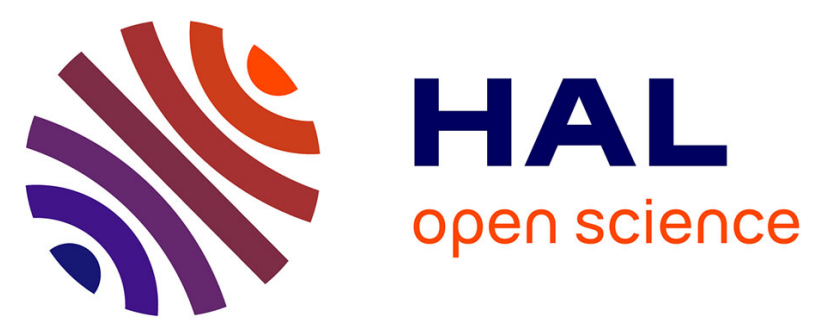

\title{
Elaboration par épitaxie en phase liquide isotherme de photopiles solaires performantes à hétérostructure graduelle GaAlAs/GaAs
}

\author{
L. Mayet, M. Gavand, D. Dutartre, A. Laugier
}

\section{To cite this version:}

L. Mayet, M. Gavand, D. Dutartre, A. Laugier. Elaboration par épitaxie en phase liquide isotherme de photopiles solaires performantes à hétérostructure graduelle GaAlAs/GaAs. Journal de Physique Lettres, 1984, 45 (17), pp.873-876. 10.1051/jphyslet:019840045017087300 . jpa-00232424

HAL Id: jpa-00232424

https://hal.science/jpa-00232424

Submitted on 1 Jan 1984

HAL is a multi-disciplinary open access archive for the deposit and dissemination of scientific research documents, whether they are published or not. The documents may come from teaching and research institutions in France or abroad, or from public or private research centers.
L'archive ouverte pluridisciplinaire HAL, est destinée au dépôt et à la diffusion de documents scientifiques de niveau recherche, publiés ou non, émanant des établissements d'enseignement et de recherche français ou étrangers, des laboratoires publics ou privés. 


\title{
Elaboration par épitaxie en phase liquide isotherme de photopiles solaires performantes à hétérostructure graduelle GaAlAs/GaAs
}

\author{
L. Mayet, M. Gavand, D. Dutartre et A. Laugier \\ Laboratoire de Physique de la Matière (*); Institut National des Sciences Appliquées de Lyon, \\ 20, avenue Albert Einstein, 69621 Villeurbanne Cedex, France
}

(Reçu le 29 mai 1984, accepté le 3 juillet 1984)

\begin{abstract}
Résumé. - Des cellules solaires performantes à structure (p) $\mathrm{Ga}_{1-x} \mathrm{Al}_{x} \mathrm{As}: \mathrm{Be}-(\mathrm{p}) \mathrm{GaAs}: \mathrm{Be}-(\mathrm{n})$ GaAs:Si sont réalisées par croissance isotherme à partir d'une solution saturée. Cette procédure simple produit à la fois une jonction diffusée dans le substrat de type $\mathrm{n}$ et une fenêtre $\mathrm{Ga}_{1-x} \mathrm{Al}_{x} \mathrm{As}$ mince $(<800 \AA)$ à gradient de bande interdite. Plusieurs structures peuvent être élaborées avec le même bain d'épitaxie.
\end{abstract}

\begin{abstract}
High efficiency (p) $\mathrm{Ga}_{1-x} \mathrm{Al}_{x} \mathrm{As}$ :Be-(p) GaAs:Be-(n) GaAs:Si solar cells are made by isothermal growth from a saturated solution. This simple procedure produces in a same time a diffused junction in the $\mathrm{n}$ type substrate and a thin $(<800 \AA)$ graded band-gap window $\mathrm{Ga}_{1-x} \mathrm{Al}_{x} \mathrm{As}$ layer. It is possible in a run to use several substrates.
\end{abstract}

\section{Introduction.}

L'épitaxie en phase liquide (E.P.L.) permet de réaliser des dispositifs optoélectroniques à base de couches minces de semiconducteurs III-V particulièrement performants grâce à la qualité des matériaux réalisés. Ainsi, dans le cas des cellules solaires en GaAs, les records de rendement sont obtenus à partir de structures élaborées par E.P.L. [1].

Cependant, l'E.P.L. souffre, par rapport aux autres techniques de croissance concurrentes (MO-CVD, MBE) d'un handicap certain dû à la difficulté de traiter de grandes surfaces et donc d'adapter ce procédé à une fabrication de masse. Cet inconvénient, qui n'est pas critique dans le cas des diodes laser par exemple en raison de leurs faibles dimensions, devient majeur pour des cellules solaires, le but étant d'obtenir des surfaces importantes, même dans le cas de dispositifs destinés à fonctionner sous forte concentration. Nous proposons, ici, une méthode simple, rapide, autorisant la réalisation de grandes surfaces au cours d'un seul processus d'épitaxie.

\section{Structure photovoltaique.}

Les photopiles GaAs à fenêtre GaAlAs sont, le plus souvent, basées sur une jonction p-n obtenue par diffusion d'un dopant de type $\mathrm{p}(\mathrm{Zn}$ ou $\mathrm{Be})$ dans le $\mathrm{GaAs}$ de type $\mathrm{n}$ au cours de la croissance du GaAlAs à partir d'un bain GaAlAs-dopant [2-5]. Ce type de jonction permet de différencier la

(*) LA, CNRS 358. 
jonction électrique de l'interface métallurgique et, ainsi, d'améliorer sa qualité. Par ailleurs, il y a création d'un gradient de concentration de porteurs favorable dans la zone $\mathrm{p}$.

La croissance de la fenêtre $\mathrm{Ga}_{1-x} \mathrm{Al}_{x} \mathrm{As}$ est, en général, obtenue par un processus classique (step-cooling, equilibrium cooling ou supercooling) conduisant à une composition en aluminium homogène à travers la couche. Son rôle principal est de diminuer la vitesse de recombinaison à la surface du GaAs. Par contre, les photons d'énergie élevée du spectre solaire y sont absorbés. La présence d'un gradient de concentration à travers cette couche, la composition en aluminium augmentant vers la surface, permet grâce au champ électrique ainsi créé de réduire les pertes dues aux recombinaisons en volume et en surface. Le champ électrique $\mathbf{E}\left(\mathbf{E}=\frac{1}{q} \frac{\mathrm{dE}_{\mathbf{g}}}{\mathrm{d} x}, E_{\mathbf{g}}\right.$ : bande interdite) ne peut être augmenté qu'en diminuant l'épaisseur de la fenêtre car la variation de la bande interdite $E_{\mathrm{g}}$ est limitée à $0,7 \mathrm{eV}$ (AlAs : $2,26 \mathrm{eV} ; \mathrm{GaAs}: 1,4 \mathrm{eV}$ ). Il faut donc réaliser une couche graduelle bien fine dont la composition en surface sera fixée par la teneur en aluminium du bain d'épitaxie. Woodall [6] a réalisé une fenêtre à gradient de concentration d'épaisseur 0,2 à $0,4 \mu \mathrm{m}$ en utilisant un bain sous-saturé. Kordos [7] en étudiant l'équilibre GaAs solide, GaAlAs liquide a obtenu des couches graduelles fines $(<1000 \AA)$ par simple contact isotherme. Il a appliqué cette technique à la réalisation d'une cellule solaire à jonction non diffusée [8].

Il nous a paru intéressant de combiner ces deux techniques et d'obtenir une structure avec deux gradients favorables : gradient de porteurs dans la zone $\mathrm{p}$, gradient de bande interdite dans la fenêtre GaAlAs (Fig. 1).

En outre, la faible épaisseur de la fenêtre $(<1000 \AA)$ doit diminuer l'absorption des photons de courte longueur d'onde dans GaAlAs.

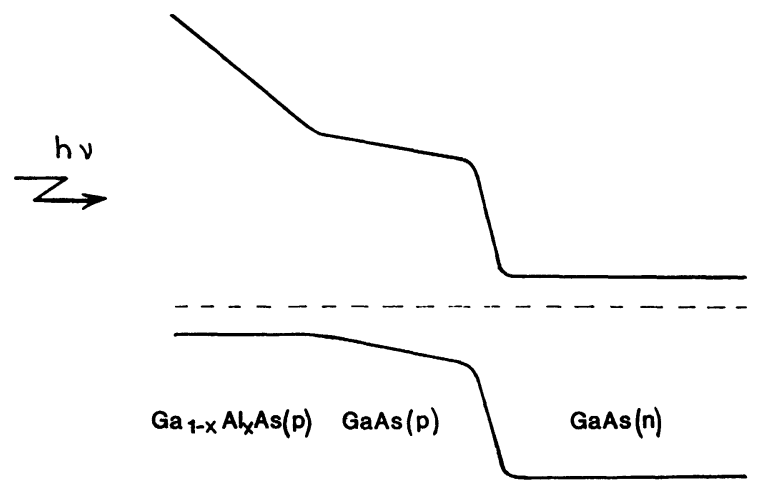

Fig. 1. - Diagramme des bandes d'énergie présentant : un gradient de porteurs dans GaAs:p; un gradient de bande interdite dans la fenêtre GaAlAs.

[Energy band diagram showing, a graded carrier concentration in p GaAs; a graded band-gap GaAlAs window.]

\section{Processus expérimental.}

La croissance a lieu dans un réacteur d'épitaxie horizontal classique avec un creuset à tiroir. Après recuit du gallium, on introduit le substrat GaAs type $\mathrm{n}\left(\langle 100\rangle, 2 \mathrm{~cm}^{2}\right)$ monocristallin soigneusement préparé et les différents composants du bain : $\mathrm{Al}, \mathrm{Be}, \mathrm{GaAs}$ polycristallin. Le bain est saturé à $800^{\circ} \mathrm{C}$ pendant 2 heures, le substrat étant protégé dans un logement du creuset pour limiter les pertes en arsenic. Puis, on amène le substrat en contact avec le bain en maintenant la température constante pendant quelques minutes, ce qui permet à la fois la diffusion du Be dans 
le substrat $\mathrm{n}$ et la croissance d'une fine couche graduelle de $\mathrm{Ga}_{1-x} \mathrm{Al}_{x} \mathrm{As}$ avec $x$ variant de 0 à l'interface à 0,85 vers la surface [7].

Le processus isotherme, la faible consommation d'Al et de $\mathrm{Be}$, la faible tension de vapeur du $\mathrm{Be}$ $\left(10^{-6}\right.$ torr à $800^{\circ} \mathrm{C}, \mathrm{Zn}: 200$ torr), permettent de considérer que le bain GaAlAs-Be n'a pas subi de variation au cours du contact. Il est donc possible de prévoir le passage d'un ou de plusieurs autres substrats sous le bain et d'obtenir ainsi une surface traitée beaucoup plus importante.

La plaquette est ensuite clivée, puis métallisée par dépôt d'un alliage Au-Ge-Ni sur le substrat de type $\mathrm{n}$ et d'un alliage Au-Be sur la fenêtre de type $\mathrm{p}$. Les contacts sont recuits sous flux d'hydrogène à $450^{\circ} \mathrm{C}$ pendant 1 minute.

\section{Résultats photovoltaïques.}

La figure 2 présente une courbe de rendement quantique normalisée typique pour une photopile à hétérostructure $\mathrm{p} \mathrm{AlGaAs} / \mathrm{p} \mathrm{GaAs} / \mathrm{n}$ GaAs à fenêtre graduelle réalisée comme décrit plus haut et dont la structure est présentée sur la figure 3. On constate l'influence de la mince fenêtre à

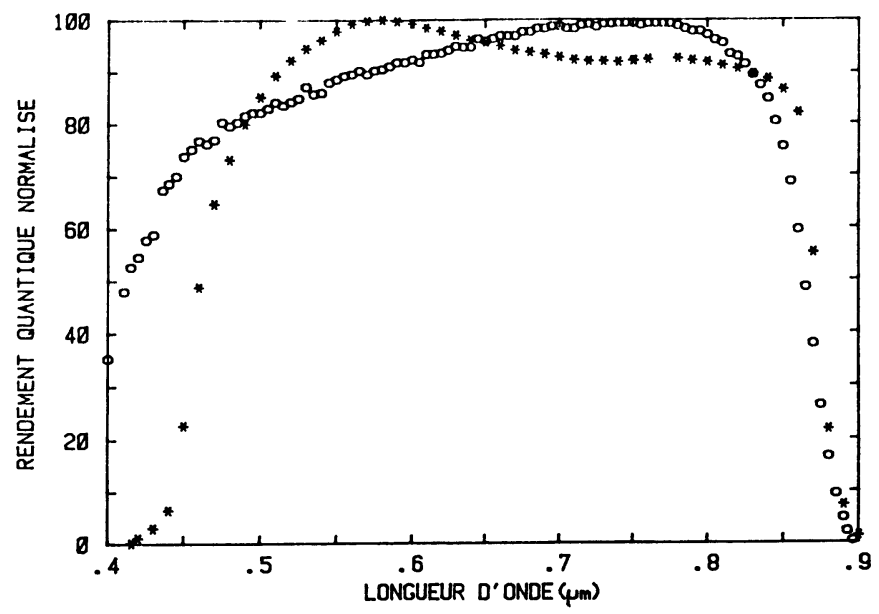

Fig. 2. - Rendement quantique normalisé de la structure représentée figure 3. $\bigcirc:$ ce travail, $*:$ structure identique mais avec une fenêtre épaisse de concentration en aluminium constante (Yoshida, Réf. [9]).

[Normalized quantum efficiency of structure represented in figure 3. $\bigcirc:$ this work, $*:$ same structure with a thick constant band-gap $\mathrm{Ga}_{0.1} \mathrm{Al}_{0.9} \mathrm{As}$ window (Yoshida, Ref. [9]).]

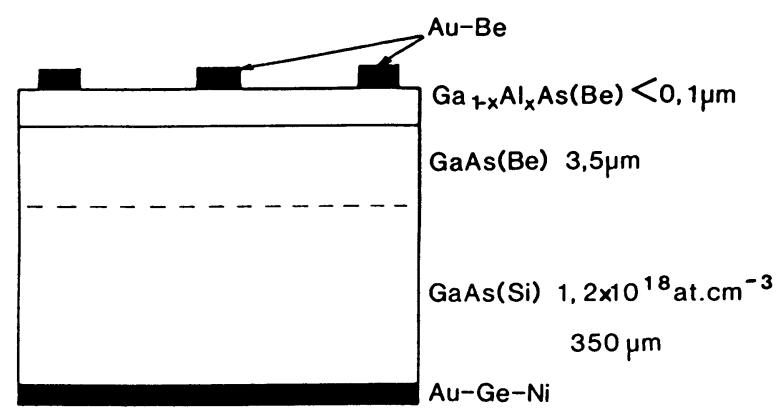

Fig. 3. - Structure d'une cellule solaire obtenue par contact isotherme d'un substrat GaAs et d'un bain GaAlAs: Be (temps de contact $10 \mathrm{~min}$ ).

[Solar cell structure made by isothermal contact of a GaAs substrate with a GaAlAs: Be melt (contact time : $10 \mathrm{~min})$.] 
bande interdite graduelle pour les photons de courte longueur d'onde par comparaison avec la réponse d'une photopile à fenêtre classique. L'amélioration provient, à la fois, du champ électrique interne qui améliore la collecte et de la réduction de l'épaisseur de la fenêtre. La valeur du champ est estimée supérieure à $5 \times 10^{4} \mathrm{~V} / \mathrm{cm}$.

Les performances du dispositif mesurées sous simulateur solaire (xénon, type AM1, $107 \mathrm{~mW} / \mathrm{cm}^{2}$ ) sont les suivantes : tension en circuit ouvert : 0,970 volt ; courant de court-circuit : $22 \mathrm{~mA} / \mathrm{cm}^{2}$ et un facteur de courbe de 0,73 sans couche anti-réflexion. Le rendement est ainsi de $14,5 \%$ (sous un soleil grille déduite). Après optimisation de la couche A.R. et de la grille collectrice, ces résultats devraient permettre de dépasser $22 \%$ sous concentration. Ainsi, par la technique décrite dans ce papier, des dispositifs photovoltaïques performants à hétérostructure basés sur le système Al-Ga-As/GaAs peuvent être réalisés en E.P.L. par une suite d'opérations technologiques simplifiées se prêtant bien à l'automatisation. Un des avantages de la méthode présentée est que la jonction, élaborée par diffusion, ne nécessite pas une préparation particulièrement délicate de la surface du substrat.

\section{Remerciements.}

Nous remercions le Laboratoire de Physique du Solide du C.N.R.S. de Bellevue pour les mesures photovoltaïques. Ce travail est soutenu par l'A.F.M.E.

\section{Bibliographie}

[1] Sahai, R., Edwall, D.D. and Harris JR, J.S., Appl. Phys. Lett. 34 (1979) 147.

[2] Woodall, J.M. and Hovel, H.J., Appl. Phys. Lett. 21 (1972) 379.

[3] Masu, K., Nakatsuka, S., Konagai, M. and Takahashi, K., J. Electrochem. Soc. 129 (1982) 1623.

[4] Flores, C. and Passoni, D., Solid State Electron. 23 (1980) 911.

[5] Imenkov, A.N., Stamkulov, A.A., Taurbaev, T.I., Tsarenkov, B.V., Shorin, V.F. and Yakovlev, Yu. P., Sov. Phys. Semicond. 12 (1978) 559.

[6] Woodall, J.M. and Hovel, H.J., Appl. Phys. Lett. 30 (1977) 492.

[7] Kordos, P., Pearson, G.L., Panish, M.B., J. Appl. Phys. 50 (1979) 6902.

[8] Kordos, P. and Pearson, G.L., Solid-State Electron. 23 (1980) 399.

[9] Yoshida, S., Mitsiui, K., Oda, T., Sogo, T., Yukimoto, Y. and Shiramata, K., 14th IEEE Photovoltaic Specialists Conf. (1980) p. 1384. 